HU-EP-02/15

\title{
DECAY CONSTANTS OF HEAVY-LIGHT MESONS IN THE RELATIVISTIC QUARK MODEL
}

\author{
D. EBERT \\ Institut für Physik, Humboldt-Universität zu Berlin, Invalidenstr.110, D-10115 Berlin, Germany \\ R. N. FAUSTOV and V. O. GALKIN \\ Institut für Physik, Humboldt-Universität zu Berlin, Invalidenstr.110, D-10115 Berlin, Germany \\ and \\ Russian Academy of Sciences, Scientific Council for Cybernetics, Vavilov Str. 40, \\ Moscow 117333, Russia
}

\begin{abstract}
The decay constants of pseudoscalar and vector heavy-light mesons are calculated in the framework of the relativistic quark model with the completely relativistic treatment of the light quark. It is argued that relativistic effects play a significant role. Good agreement of the model predictions with recent lattice and QCD sum rule calculations as well as available experimental data is found.
\end{abstract}

The pseudoscalar and vector decay constants of the heavy-light mesons are important hadronic characteristics of these mesons. They determine such quantities as the weak decay rates of mesons to lepton pairs and the magnitude of $B_{d}-\bar{B}_{d}$ and $B_{s}-\bar{B}_{s}$ mixings. These constants also enter the nonleptonic decay rates, considered in the factorization approximation. In this brief note we consider them in the framework of the relativistic quark model.

The decay constants $f_{P}$ and $f_{V}$ of the pseudoscalar $(P)$ and vector $(V)$ mesons parameterize the matrix elements of the weak current between the corresponding meson and the vacuum. In the case of a meson, composed from a heavy antiquark $\bar{Q}$ and a light quark $q$, they are defined by

$$
\begin{aligned}
& \left\langle 0\left|\bar{Q} \gamma^{\mu} \gamma_{5} q\right| P(\mathbf{K})\right\rangle=i f_{P} K^{\mu}, \\
& \left\langle 0\left|\bar{Q} \gamma^{\mu} q\right| V(\mathbf{K}, \varepsilon)\right\rangle=f_{V} M_{V} \varepsilon^{\mu}
\end{aligned}
$$


where $\mathbf{K}$ is the meson momentum, $\varepsilon$ and $M_{V}$ are the polarisation vector and mass of the vector meson.

In the relativistic quark model the decay constants can be expressed through the meson wave function $\Phi_{P, V}(p)$ in the momentum space and are given by녈

$$
\begin{aligned}
f_{P, V}= & \sqrt{\frac{12}{M_{P, V}}} \int \frac{d^{3} p}{(2 \pi)^{3}}\left(\frac{\epsilon_{q}(p)+m_{q}}{2 \epsilon_{q}(p)}\right)^{1 / 2}\left(\frac{\epsilon_{Q}(p)+m_{Q}}{2 \epsilon_{Q}(p)}\right)^{1 / 2} \\
& \times\left\{1+\lambda_{P, V} \frac{\mathbf{p}^{2}}{\left[\epsilon_{q}(p)+m_{q}\right]\left[\epsilon_{Q}(p)+m_{Q}\right]}\right\} \Phi_{P, V}(p),
\end{aligned}
$$

with $\lambda_{P}=-1$ and $\lambda_{V}=1 / 3$. In the nonrelativistic limit $p^{2} / m^{2} \rightarrow 0$ these expressions for decay constants give the well-known formula

$$
f_{P}^{\mathrm{NR}}=f_{V}^{\mathrm{NR}}=\sqrt{\frac{12}{M_{P, V}}}\left|\Psi_{P, V}(0)\right|,
$$

where $\Psi_{P, V}(0)$ is the meson wave function at the origin $r=0$. In the case of the heavy-light $B$ and $D$ mesons, considered in the infinitely heavy quark mass limit $m_{Q} \rightarrow \infty$, the meson wave function is determined by the light quark only. Therefore in this limit, as it follows from Eq. (3), the decay constants scale inversely proportional to the square root of the meson mass: $f_{P} \sqrt{M_{P}}=$ const. This leads to the relation $f_{B}^{\mathrm{NR}} / f_{D}^{\mathrm{NR}}=\sqrt{M_{D} / M_{B}}$ between $B$ and $D$ decay constants. However, the relativistic effects break down this scale behaviour. Expanding expression (3) in $1 / m_{Q}$, we get

$$
\begin{aligned}
& \frac{f_{B}}{f_{D}}= \sqrt{\frac{M_{D}}{M_{B}}}\left\{1+\frac{1}{2}\left(\frac{1}{m_{c}}-\frac{1}{m_{b}}\right) \frac{\int \frac{d^{3} p}{(2 \pi)^{3}} \frac{\mathbf{p}^{2}}{\sqrt{2 \epsilon_{q}(p)\left[\epsilon_{q}(p)+m_{q}\right]}} \Phi_{P}(p)}{\int \frac{d^{3} p}{(2 \pi)^{3}}\left(\frac{\epsilon_{q}(p)+m_{q}}{2 \epsilon_{q}(p)}\right)^{1 / 2} \Phi_{P}(p)}\right. \\
&\left.+O\left(\frac{1}{m_{Q}^{2}}\right)\right\} \approx \sqrt{\frac{M_{D}}{M_{B}}}\left\{1+0.20+O\left(\frac{1}{m_{Q}^{2}}\right)\right\} .
\end{aligned}
$$

Thus we see that the first order corrections in $1 / m_{Q}$ shift the ratio of the pseudoscalar decay constants of $D$ and $B$ mesons by about $20 \%$. As a result, relativistic values of $f_{B}$ and $f_{D}$ are closer to each other than nonrelativistic ones. As it will be seen later, this fact is important for bringing quark model predictions in agreement with recent lattice and QCD sum rule calculations.

The calculated values of the pseudoscalar and vector decay constants of heavylight mesons in our model are displayed in Table 1. In the first row we show nonrelativistic predictions and in the second row relativistic results (3). For these calculations we used the wave functions of the heavy-light mesons obtained in the relativistic quark model based on the quasipotential approach in quantum field theory in calculating their mass spectra $\mathrm{B}^{\text {. It }}$ is important to stress that the light quark 
Table 1. Pseudoscalar and vector decay constants of mesons with open flavour (in $\mathrm{MeV}$ ).

\begin{tabular}{ccccccccccc}
\hline Meson & $D$ & $D^{*}$ & $D_{s}$ & $D_{s}^{*}$ & $B$ & $B^{*}$ & $B_{s}$ & $B_{s}^{*}$ & $B_{c}$ & $B_{c}^{*}$ \\
\hline$f^{\mathrm{NR}}$ & 332 & 332 & 384 & 384 & 213 & 213 & 278 & 278 & 562 & 562 \\
$f_{P, V}$ & 243 & 315 & 266 & 335 & 178 & 195 & 196 & 214 & 433 & 503 \\
\hline
\end{tabular}

was treated completely relativistically, without employing an unjustified expansion in inverse powers of the light quark mass. Only the heavy quark expansion was used in order to simplify calculations. The relativistic treatment of the light quark has a significant impact on the wave functions and, as a result, on the decay constants of the heavy-light mesons. It increases the ratio $f_{B} / f_{D}$ from 0.64 in the nonrelativistic limit to 0.74 and decreases the ratios $f_{B_{s}} / f_{B}$ from 1.3 to 1.1 and $f_{D_{s}} / f_{D}$ from 1.2 to 1.1 , respectively.

Table 2. Pseudoscalar decay constants of the heavy-light mesons (in $\mathrm{MeV}$ ). Comparison of our results with averaged lattice data, QCD sum rule predictions and averaged experimental data.

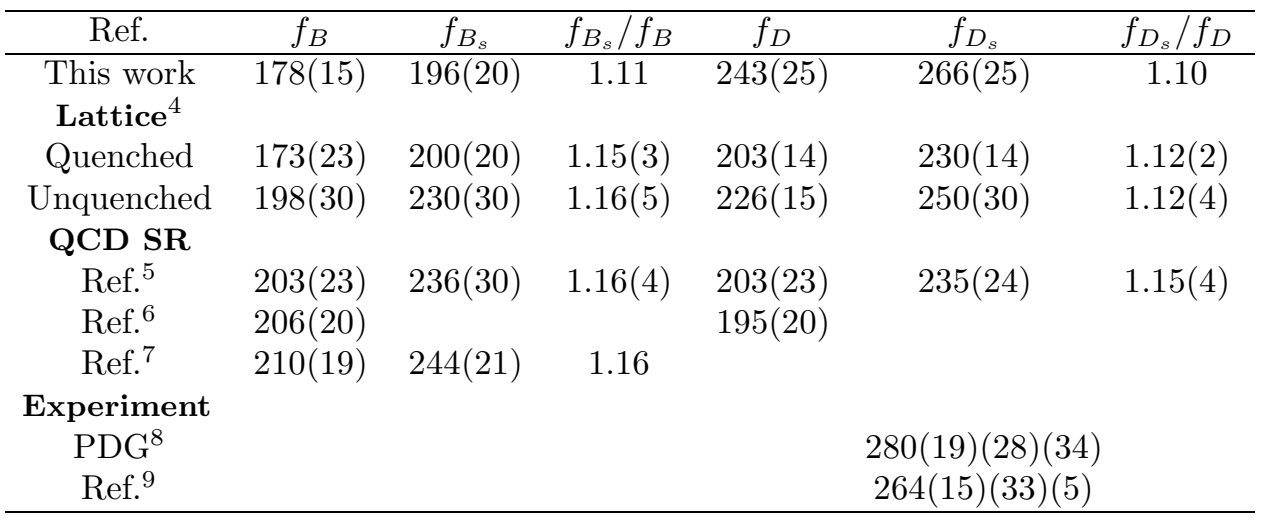

In Table 2 we compare our predictions ${ }^{a}$ for pseudoscalar decay constants of the heavy-light mesons as well as their ratios with other theoretical results and available experimental data. The pseudoscalar decay constants of $B$ and $D$ mesons were studied rather extensively in different theoretical approaches. In this Table we present the averaged lattice data obtained both in quenched and unquenched approximations from the recent reviewt. We also list the most recent QCD sum rule predictions 6 6. Reliable experimental data at present exist only for $f_{D_{s}}$, which was measured by several experimental collaborations (ALEPH, DELPHI, L3, OPAL, Beatrice, CLEO, E653, WA75, BES) both in the $D_{s} \rightarrow \mu \nu_{\mu}$ and the $D_{s} \rightarrow \tau \nu_{\tau}$ decay channels. Unfortunately, experimental errors are still large. In Table 2 we present two experimental averages: one from PDGl and the other from the recent

${ }^{a}$ We roughly estimate the error in our calculations (mainly coming from the wave functions) to be about $10 \%$. 
experimental review 2 . There exists also the first experimental value for $f_{D^{+}}=$ $300_{-150-40}^{+180+80} \mathrm{MeV}$, but based on only one $D^{+} \rightarrow \mu^{+} \nu_{\mu}$ event 10 and thus with very large errors. We see from Table 2 that there is good (within error bars) agreement between all presented theoretical predictions as well as with available experimental data. However, the QCD sum rules seem tend to prefer for the $f_{B} / f_{D}$ ratio the value close to 1 , while somewhat smaller values are favoured by the lattice $(0.85)$ and by our model (0.74).

We can also compare our prediction for the vector decay constant $f_{B^{*}}$ with the recent lattice calculation in quenched approximation 11 :

$$
f_{B^{*}}=177 \pm 6 \pm 17 \mathrm{MeV} \text { and } f_{B^{*}} / f_{B}=1.01 \pm 0.01_{-0.01}^{+0.04}
$$

The ratios of vector and pseudoscalar decay constants $f_{V} / f_{P}$ are also predicted by heavy quark effective theory 12 (HQET)

$$
f_{B^{*}} / f_{B} \cong 1.07 \pm 0.03, \quad f_{D^{*}} / f_{D} \cong 1.31 \pm 0.08
$$

For these ratios in our model we have

$$
f_{B^{*}} / f_{B} \cong 1.09, \quad f_{D^{*}} / f_{D} \cong 1.30
$$

in good agreement with both lattice and HQET results.

In summary, we calculated the decay constants of the pseudoscalar and vector heavy-light mesons in the framework of the relativistic quark model paying special attention to the complete relativistic treatment of the light quark. It was found that the relativistic effects significantly reduce the values of the decay constants and violate the scaling behaviour with masses of the decay constants which emerge in the nonrelativistic and heavy quark limits. As a result the ratio $f_{B} / f_{D}$ is considerably increased bringing relativistic quark model predictions in agreement with recent lattice and QCD sum rule calculations.

The authors express their gratitude to A. Ali Khan, M. Müller-Preussker and V. Savrin for support and discussions. Two of us (R.N.F and V.O.G.) were supported in part by the Deutsche Forschungsgemeinschaft under contract Eb 139/2-1, Russian Foundation for Fundamental Research under Grant No. 00-02-17768 and Russian Ministry of Education under Grant No. E00-3.3-45.

\section{References}

1. V. O. Galkin, A. Yu. Mishurov and R. N. Faustov, Sov. J. Nucl. Phys. 53, 1026 (1991) [Yad. Fiz. 53, 1676 (1991)].

2. S. Godfrey, Phys. Rev. D 32, 189 (1985).

3. D. Ebert, V. O. Galkin and R. N. Faustov, Phys. Rev. D 57, 5663 (1998); Erratum 59, 019902 (1999).

4. S. Ryan, Nucl. Phys. B (Proc. Suppl.) 106, 86 (2002).

5. S. Narison, hep-ph/0202200.

6. A. A. Penin and M. Steinhauser, Phys. Rev. D 65, 054006 (2002). 
7. M. Jamin and B. O. Lange, Phys. Rev. D 65, 056005 (2002).

8. Particle Data Group, D. E. Groom et al., Eur. Phys. J. C 15, 1 (2000).

9. S. Söldner-Rembold, talk at HEP2001, hep-ex/0109023.

10. J. Z. Bai et al. (BES Collaboration), Phys. Lett. B 429, 188 (1998).

11. C. Bernard et al., Phys. Rev. D 65, 014510 (2002).

12. M. Neubert, Phys. Rev. D 46, 1076 (1992). 